\title{
Preliminary results of electrical characterization of GO towards MCF7 and MCF10a at different concentrations
}

\begin{abstract}
GO is the 2D carbon sheet with additional functional groups, is more stable in various solvents, easy to be produced and manipulated especially in biological system. At the moment, GO is only utilized as the drug delivery agent during treatment. In this study, the resistivity of GO towards breast cancer cell (MCF7) and normal breast cell (MCF10a) using interdigitated electrodes (IDE) were investigated. The interaction of different concentrations of GO as the sensing material on the tested cells which act as analyte can change electrical response. The tested cell were treated with six different concentrations of GO and was dropped to the IDE with different period of time in order to examine electrical behavior. For MCF10a, at high concentration the resistances of MCF10 remain in the same order of magnitude with increasing time of detection while for MCF7 at high concentration, the resistances were greatly influenced by the time of detection where the value significantly changed after 5 minutes and 10 minutes. The number of viable cell does not give effect to the resistance.
\end{abstract}

Keyword: Graphene oxide; Cancer cell; Concentration; Size of flakes; MCF10a; MCF7 